\title{
Preface for the special issue on argument strength
}

\author{
Gabriella Pigozzi ${ }^{a}$ and Srdjan Vesic ${ }^{\text {b }}$ \\ ${ }^{a}$ LAMSADE, Université Paris-Dauphine, PSL Research University, 75016 Paris, France \\ E-mail: gabriella.pigozzi@dauphine.fr \\ ${ }^{\mathrm{b}}$ CRIL - CNRS, Univ. Artois, Lens, France \\ E-mail:vesic@cril.fr
}

Arguments may vary in strength. The strength of an argument is influenced by different factors like the plausibility of its premises or the nature of the link between its premises and conclusion. The first Argument Strength workshop was organized in 2016 at Ruhr-University Bochum (Germany). The goal was to gather researchers to study different questions such as the factors that influence the strength of an argument, different formal representations of argument strength, propagation of argument strength with respect to sub-arguments, strength in the context of argument accrual, the link between argument strength and argument specificity, the link between formal and informal approaches to argument strength, and how the preferences on premises influence the evaluation of arguments. The selected papers of the first workshop were published in a special issue of the Journal of Applied Logics - IfCoLog Journal, Volume 5 Number 3, 2018.

To pursue the study on those questions, the second Argument Strength workshop was organized in 2018 at IRIT Toulouse (France). This special issue contains the revised versions of some of the papers selected from the contributions presented at that workshop as well as new submissions received after the workshop. In total, we have received seven submissions and each of them has been carefully reviewed by three external reviewers. We wish to thank the reviewers for their generous help, time and expertise.

Here is a short overview of the four papers accepted for publication.

Heyninck and Straßer [3] point out that ASPIC + in its general form does not satisfy some rationality postulates (e.g. non-interference). They also underline that the links between ASPIC + and other forms of reasoning are not sufficiently studied. In this paper, they study a fragment of ASPIC+ without undercut and show that it satisfies the rationality postulates for structured argumentation from the literature under stable and preferred semantics. They also prove results regarding the links between this fragment of ASPIC+ and reasoning with maximal consistent sets.

Bonzon et al. [1] study the applicability of existing ranking-based semantics when it comes to modelling persuasion. They note that some existing principles for ranking-based semantics (like void precedence) are not compatible with other intuitive requirements that a semantics for modelling persuasion dialogues should satisfy (like procatalepsis and the fading principle). They propose a parametric rankingbased semantics, whose parameter allows, among other things, to control the behaviour of the semantics and thus to which extent it satisfies which principle. 
A new framework for representing the strength of arguments called Strength-based Argumentation Framework (StrAF) is proposed by Rossit et al. [4]. Their proposal is based on Dung-style abstract argumentation, by associating an integer weight to each argument, that can also combine their attacking strengths (accrual) and allow comparison of the arguments' respective strengths. The paper defines two kinds of semantics (strong and weak) considering the accrual of arguments. The authors then show that their semantics are proper generalizations of Dung semantics. Finally, they provide a complexity analysis for strong and weak stable semantics as well as an encoding as pseudo-boolean constraint satisfaction problem.

Cohen et al. [2] propose an argumentation formalism that models reasoning in a multi-agent setting where agents can exchange object-level information and take into account reasons for or against the credibility of other agents (the informants). In their approach, knowledge representation and reasoning are inspired by Defeasible Logic Programming (DeLP). Agents share tentative information in the form of defeasible rules and each agent can obtain information from other agents that have different degrees of credibility. An agent has a preference relation over its informants and this preference relation is key to define a notion of the relative strength of arguments. In that way, agents can argue about the arguments' strength by providing arguments for or against their informants. The authors show their defeasible reasoning machinery is sound, preventing an agent from warranting contradicting conclusions.

\section{References}

[1] E. Bonzon, J. Delobelle, S. Konieczny and N. Maudet, A parametrized ranking-based semantics compatible with persuasion principles, Argument \& Computation 12(1) (2021), 49-85. doi:10.3233/AAC-200905.

[2] A. Cohen, S. Gottifredi, L.H. Tamargo, A.J. García and G.R. Simari, An informant-based approach to argument strength in defeasible logic programming, Argument \& Computation 12(1) (2021), 115-147. doi:10.3233/aac-200902.

[3] J. Heyninck and C. Straßer, Rationality and maximal consistent sets for a fragment of ASPIC + without undercut, Argument \& Computation 12(1) (2021), 3-47. doi:10.3233/aac-200903.

[4] J. Rossit, J.-G. Mailly, Y. Dimopoulos and P. Moraitis, United we stand: Accruals in strength-based argumentation, Argument \& Computation 12(1) (2021), 87-113. doi:10.3233/aac-200904. 